\title{
Répartie à propos de l'article de Philippe Lysy
}

> Répartie à l'article de Philippe A. Lysy - La thérapie cellulaire du diabète : le point sur les actualités (médecine/sciences, 2016, vol. 32, $n^{\circ} 4$, pages $401-7$ ). <

Nous souhaiterions apporter quelques précisions après la lecture de l'article de Philippe Lysy, paru dans le numéro d'avril 2016 de $\mathrm{m} / \mathrm{s}$ [1], notamment en ce qui concerne le risque tumoral associé à l'utilisation clinique de précurseurs dérivés de cellules souches embryonnaires humaines (CSEh) ou pluripotentes induites (iPS). La rédaction du texte pourrait induire une interprétation erronée des données mentionnées. II n'y est en effet pas toujours clair de distinguer si le propos de l'auteur concerne l'ensemble des précurseurs dérivés de CSEh, ou les seuls précurseurs $\beta$ pancréatiques qui sont l'objet de l'article.

1. Dans le résumé, le «risque carcinogénique »[ des cellules souches pluripotentes humaines] est qualifié d'《 actuellement inévitable » et il est également mentionné dans le corps du texte que "L'enthousiasme généré par ces études in vivo a été obscurci par la démonstration de la propension des CSE à former des tératomes par prolifération et différenciation incontrôlée des populations cellulaires indifférenciées. Le risque repose sur la transplantation de CSE ayant accumulé, au décours de cycles de prolifération in vitro, des anomalies chromosomiques conférant un risque de cancérisation ».

Dans la mesure où plusieurs essais cliniques sont en cours depuis 2010 avec des précurseurs dérivés de CSEh (voir ci-dessous), il nous semble important d'apporter deux précisions :

(1) Aucun protocole clinique ne transplante de CSEh sous une forme indifférenciée; seuls des progéniteurs issus de ces CSEh et irréversiblement engagés dans une voie de différenciation sont utilisés.

(2) Le risque tumoral a pour origine deux mécanismes distincts : d'une part le statut pluripotent des cellules indifférenciées, indépendamment de la présence éventuelle de mutations génétiques acquises lors de leur culture. La formation de tératomes, tumeurs bénignes, est une propriété intrinsèque de toute cellule souche pluripotente, qui sert d'ailleurs à affirmer la pluripo-

\section{Laure Coulombel}

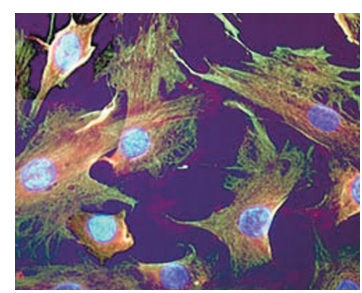

DR Inserm émérite, Paris, France. laure.coulombel@inserm.fr

tence. D'autre part, les CSEh peuvent acquérir, lors de leur dérivation et de leur culture, de possibles mutations génétiques. Celles-ci se transmettront à leur descendance différenciée, et seront source d'instabilité génétique potentiellement inductrice de tumeurs malignes. Ces deux risques sont évalués et minimisés, d'une part, par la sélection des cellules thérapeutiques obtenues via la différenciation des CSEh en progéniteurs différenciés et par l'analyse d'une contamination éventuelle des produits thérapeutiques finaux par des cellules pluripotentes résiduelles, et, d'autre part, par l'analyse génomique rigoureuse des CSEh initiales et des produits cellulaires finaux utilisés en clinique (voir recommandations de l'ISSCR [2]).

Un protocole de différenciation efficace des CSEh, en permettant l'obtention d'une proportion importante de cellules du phénotype désiré, réduira la contamination potentielle par des cellules indifférenciées et facilitera la purification des précurseurs thérapeutiques.

2. L'auteur fait allusion aussi bien aux CSEh qu'aux iPS ; il est donc nécessaire de distinguer le risque associé à l'utilisation de précurseurs dérivés de CSEh, cellules non manipulées génétiquement, et celui associé aux dérivés d'iPS, cellules qui ont subi un processus brutal et artificiel de reprogrammation épigénétique, dont les conséquences sur le devenir à long terme des cellules et leur stabilité génétique sont encore mal connues. Il est important de mentionner qu'aujourd'hui, en 2016, cette reprogrammation est induite non seulement sans recourir à l'expression d'oncogènes (dont cmyc) - utilisés initialement en 2007 -, mais également sans faire intervenir des vecteurs intégratifs véhiculant les facteurs de reprogrammation [3, 4].

3. Les essais cliniques - 12 sont en cours - avec des précurseurs dérivés de CSEh et qui ont démarré en 2010 n'ont pas révélé d'effets secondaires indésirables, en particulier pas de développement tumoral. II faut rappeler que les essais les plus importants par le nombre de patients inclus concernent deux pathologies de la rétine, la dégénérescence maculaire liée à l'âge (DMLA) et la dystrophie héréditaire maculaire de type Stargardt's, d'origine génétique [5]. Six essais sont en cours dans 4 pays, États-Unis, Royaume-Uni, Corée du Sud et Israël, concernant environ 80 patients, et les données disponibles ont été publiées pour 22 patients $[6,7]$. Trois autres essais en cours concernent d'autres pathologies : traumatismes de la moelle épinière 
(société Geron puis Asterias, États-Unis [8]), insuffisance ventriculaire gauche post-ischémique ( $P r$ Philippe Menasché, APHP, France [9]) et diabète (société Viacyte, États-Unis, essai mentionné par P. Lysy). Viacyte utilise un dispositif d'encapsulation des précurseurs endodermiques, dont il faut préciser qu'il minimise les risques non seulement tumoraux mais aussi immunologiques [10].

$\varepsilon n$ revanche, le seul essai clinique utilisant des précurseurs rétiniens dérivés d'iPS autologues qui avait débuté en 2014 (Japon) a été arrêté en partie en raison de l'observation d'anomalies génétiques dans les précurseurs devant être greffés. II devrait reprendre, mais avec des cellules dérivées d'iPS allogéniques provenant de biobanques.

4. Les CSEh utilisées dans ces essais cliniques sont généralement des lignées de cellules validées et parfaitement caractérisées depuis de nombreuses années; l'assertion selon laquelle l'utilisation clinique des CSEh requiert la "généralisation de l'utilisation d'embryons humains » (page 403) apparaît contestable.

5. Enfin, nous renvoyons le lecteur au numéro thématique « Diabète : approches thérapeutiques émergentes » comprenant 14 synthèses et publié par $\mathrm{m} / \mathrm{s}$ en août/septembre 2013, et en particulier aux articles [11, 12] traitant de l'utilisation de cellules $\beta$ pancréatiques dérivées de cellules souches pluripotentes ou de protocoles de transdifférenciation. $\diamond$

Repartee about Philippe Lysy's article

\section{REMERCIEMENTS}

Merci à Philippe Ménasché de sa relecture.

\section{LIENS D'INTÉRÊT}

L'auteur déclare n'avoir aucun lien d'intérêt concernant les données publiées dans cet article.

\section{RÉFÉRENCES}

1. Lysy P. La thérapie cellulaire du diabète: le point sur les actualités. Med Sci (Paris) $2016 ; 32: 401-7$.

2. International Societey for stem cell research. Guidelines for stem cell research and clinical trasnlation. May 2016. http://www.isscr.org/docs/ default-source/guidelines/isscr-guidelines-for-stem-cell-research-andclinical-translation.pdf?sfvrsn $=2$

3. Wu SM, Hochedlinger K, Harnessing the potential of induced pluripotent stem cells for regenerative medicine. Nat Cell Biol $2011 ; 13: 497-505$.

4. Trounson A, DeWitt ND. Pluripotent stem cells progressing to the clinic. Nat Rev Mol Biol 2016 ; 17 : 194-200.

5. Kimbrel \&A, Lanza R. Current status of pluripotent stem cells: moving the first therapies to the clinic. Nat Rev Drug Discov 2015 ; 14 : 681-92.

6. Schwartz SD, Tan G, Hosseini H, Nagiel A. Subretinal transplantation of embryonic stem cell-derived retinal pigment epithelium for the treatment of macular degeneration: an assessment at 4 years. Invest Ophthalmol Vis Sci $2016 ; 57$ : ORSFcl-9.

7. Song WK, Park KM, Kim HJ, et al. Treatment of macular degeneration using embryonic stem cell-derived retinal pigment epithelium: preliminary results in Asian patients. Stem Cell Reports $2015 ; 4: 860-72$.

8. Lebkowski JS. Phase I clinical trial of human embryonic stem cell derived oligodendrocyte progenitors in patients with neurologically complete thoracic spinal cord injury: results and next steps. American Society for Cell and Gene Therapy conference 2014 (http://asteriasbiotherapeutics.com/ pipeline/ast-opcl/).

9. Menasché $P$, Vanneaux $V$, Hagège $A$, et al. Human embryonic stem cellderived cardiac progenitors for severe heart failure treatment: first clinical case report. Eur Heart J 2015 ; $36: 2011-7$.

10. Agulnick AD, Ambruzs DM, Moorman MA, et al. Insulin-producing endocrine cells differentiated in vitro from human embryonic stem cells function in macroencapsulation devices in vivo. Stem Cells Transl Med $2015 ; 4$ : 1214-22.

11. Kunjom Mfopou J, Bouwens L. Différenciation des cellules souches pluripotentes en cellules pancréatiques. Med Sci (Paris) 2013 ; 29 : 736-43.

12. Vieira A, Druelle N, Courtney M, et al. Reprogrammation des cellules pancréatiques en cellules $\beta$. Med Sci (Paris) 2013 ; 29 : 749-55.

\section{TIRÉS À PART}

L. Coulombel

\section{LA FONDATION PREMUP : UN OPÉRATEUR DE TERRAIN EN PÉRINATALITÉ RECONNU POUR SON EXCELLENCE ET SON INTERDISCIPLINARITÉ}

La Fondation de coopération scientifique PremUp, unique en Europe, intervient sur la prévention du handicap à la naissance, par la protection de la santé de la femme enceinte et du nouveau-né.
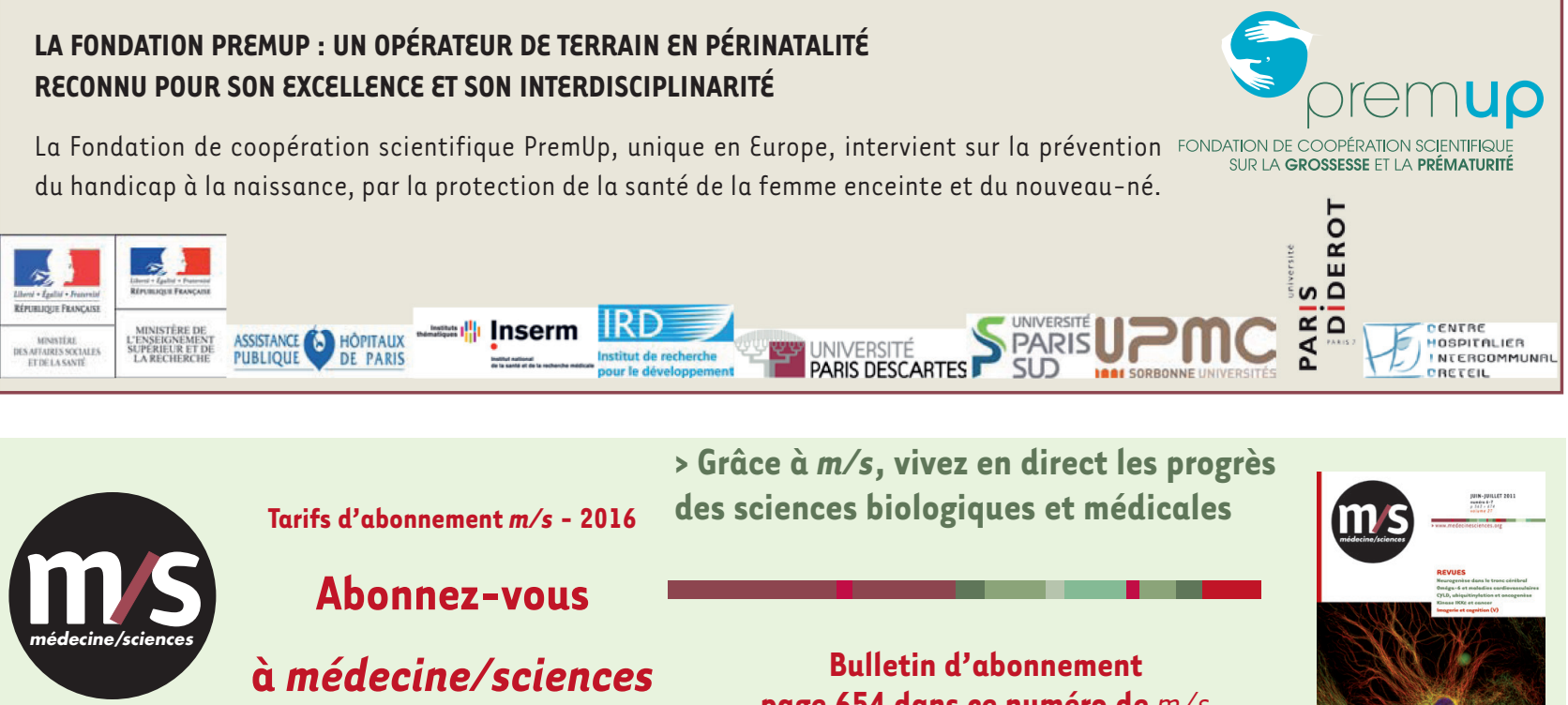

Tarifs d'abonnement $m / s-2016$

$>$ Grâce à $\mathrm{m} / \mathrm{s}$, vivez en direct les progrès

des sciences biologiques et médicales

Abonnez-vous

à médecine/sciences

Bulletin d'abonnement page 654 dans ce numéro de $\mathrm{m} / \mathrm{s}$

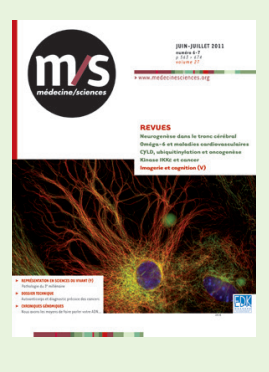

